\title{
Janz Syndrome (Youth Mioclonics), Two Brothers Case Report and Literature Review
}

\author{
José Roberto Cárdenas Félix ${ }^{1}$, Humberto Ortiz Castañeda ${ }^{2}$ and Gabriel Miranda Nava ${ }^{3 *}$ \\ ${ }^{1}$ Undergraduate intern doctor Regional Military Hospital of specialties of Guadalajara, Mexico \\ ${ }^{2}$ Director, Regional Military Hospital of specialties of Guadalajara, Mexico \\ ${ }^{3}$ Neurologist, Neurology Service of the Regional Military Hospital of specialties of Guadalajara, Mexico
}

*Corresponding author: Gabriel Miranda Nava, Neurologist, Neurology Service of the Regional Military Hospital of specialties of Guadalajara, Mexico.

\begin{abstract}
Introduction: Juvenile myoclonic epilepsy classified as a generalized genetic epilepsy, it occurs with sudden jerks of the upper limbs and if the lower limbs are affected, with falls and without loss of consciousness. They occur preferably in the first minutes upon waking. They are triggered by sleep deprivation and alcohol intake. The disease follows a non-progressive course.

Clinical Case: A 15-year-old female patient presented to the service due to an absence crisis and an apparent insomnia problem. The semiology of absence crisis is interrogated and in effect, as well as occasional nocturnal myoclonus, an electroencephalogram is performed and a $4 \mathrm{~Hz}$ slow wave poly tip pattern is found throughout the stroke, especially in photo stimulation (Photo 1,2). The patient has an older brother of 17 years who has developed school problems without a previous diagnosis of epilepsy, electroencephalogram is performed and also has a generalized slow-wave poly tip pattern (Photo 3,4) Valproate treatment is initiated with good response.
\end{abstract}

Conclusion: Juvenile myoclonic epilepsy forms at least one tenth of the epileptic syndromes, of a genetic nature with a family presentation that presents with repetitive abrupt movements. Valproic acid is a first-choice treatment and lifestyle changes and their triggers.

Keywords: Epilepsy; Juvenile myoclonic epilepsy; Janz; Myoclonus

\section{Introduction}

Juvenile myoclonic epilepsy, also known as Janz disease, is classified as a generalized genetic epilepsy, with sudden jerking of upper limbs with projection of the object in their hands, and if the lower limbs are occasionally affected, with fall, presentation with crisis Generalized tonic-clonic may also occur, and less frequently absences. There is no loss of consciousness. They occur preferably in the first minutes upon waking. They are triggered by sleep deprivation and alcohol intake. Patients with JIA do not usually have intellectual or neurological deficits and the disease follows a non-progressive course [1].

The objective is to present a clinical case of familial Janz epilepsy and review of the literature.

\section{Epidemiology}

It occurs in 5 to $10 \%$ of epileptic syndromes. Epilepsy of genetic origin, with marker on the short arm of chromosome 6p21.3 and has also been identified on the long arm of chromosome 15 (15q14) occurs in both sexes and starting range between 8 and 26 years, with family history of Idiopathic Generalized Epilepsy in 25\% of cases. With presentation of 1 in every 1000-2000 [2].

\section{Clinical Presentation}

Reiterating the starting range of 8-26 years with an average presentation at 13 years of age, it presents myoclonus which are characterized by single or repetitive, abrupt, arrhythmic and involuntary shaking of predominance in the upper limb being symmetrical, however, it may appear Unilateral are short, with an approximate duration of one second which varies from violent movements to minimal contractions.

It is possible that there is a case where there are no visible movements, however the patient refers to a sensation related to electric shock inside the body. 


\section{Clinical Case}

A 15-year-old female patient presented to the service due to an absence crisis and an apparent insomnia problem. The semiology of absence seizures is interrogated and in effect, as well as occasional nocturnal myoclonus, electroencephalogram is performed and a 4-wave slow polypoint pattern generalized throughout the stroke is found, especially in photo stimulation (Figure 1,2).

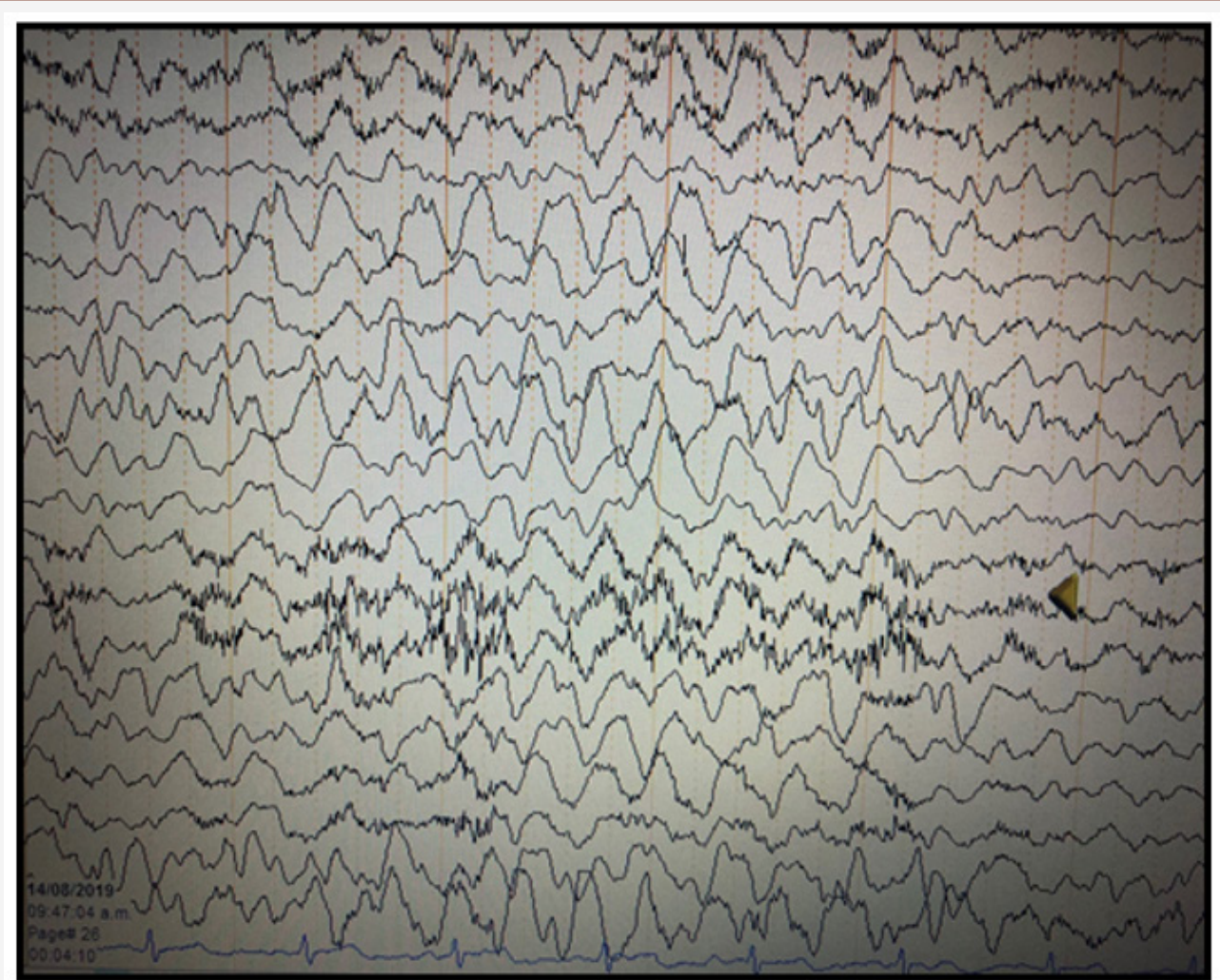

Figure 1

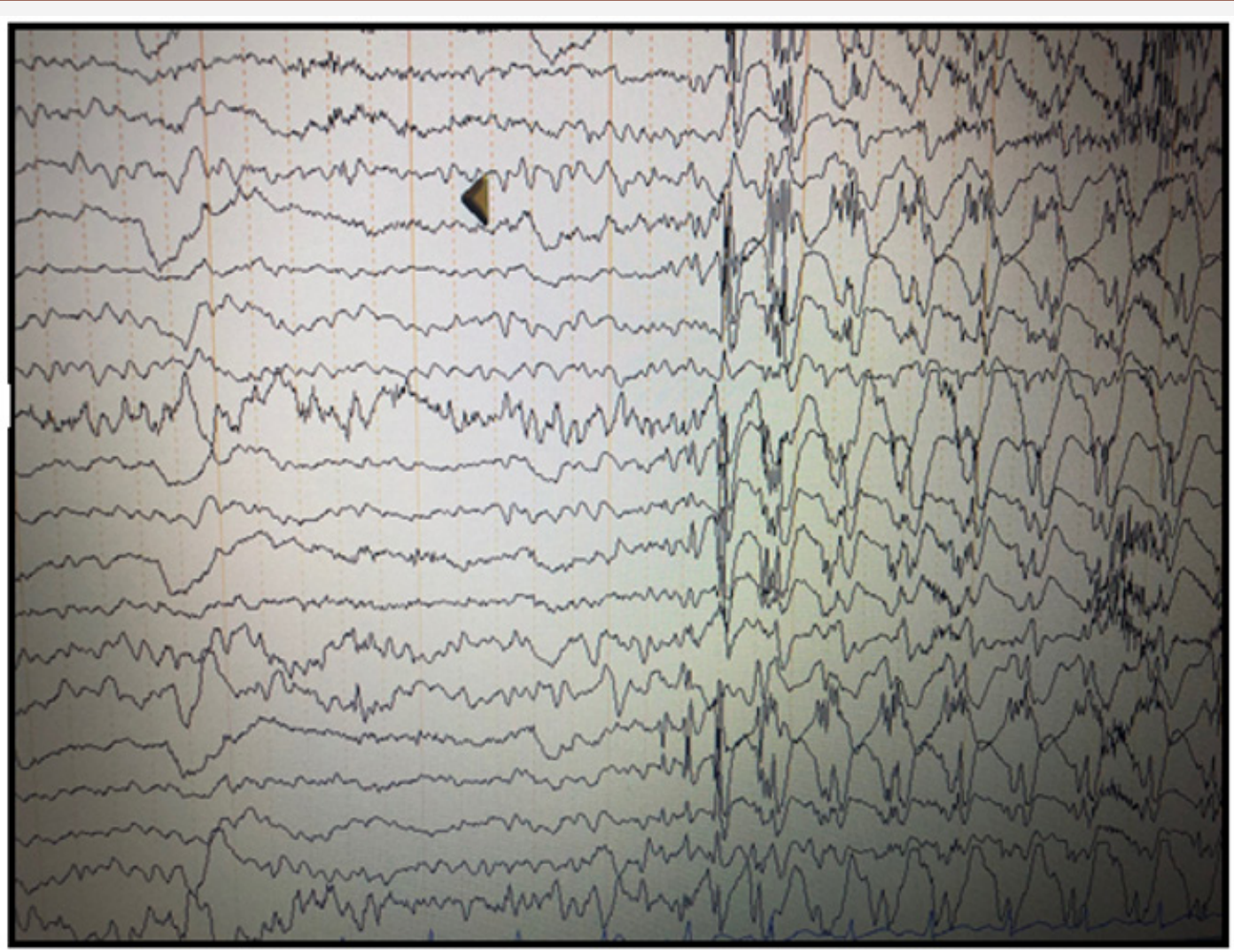

Figure 2 
The patient has an older brother of 17 years who has developed school problems without a previous diagnosis of epilepsy, electroencephalogram is performed and also presents a generalized slow wave polypoon pattern (Figure 3,4). Valproate treatment is initiated with a good response.

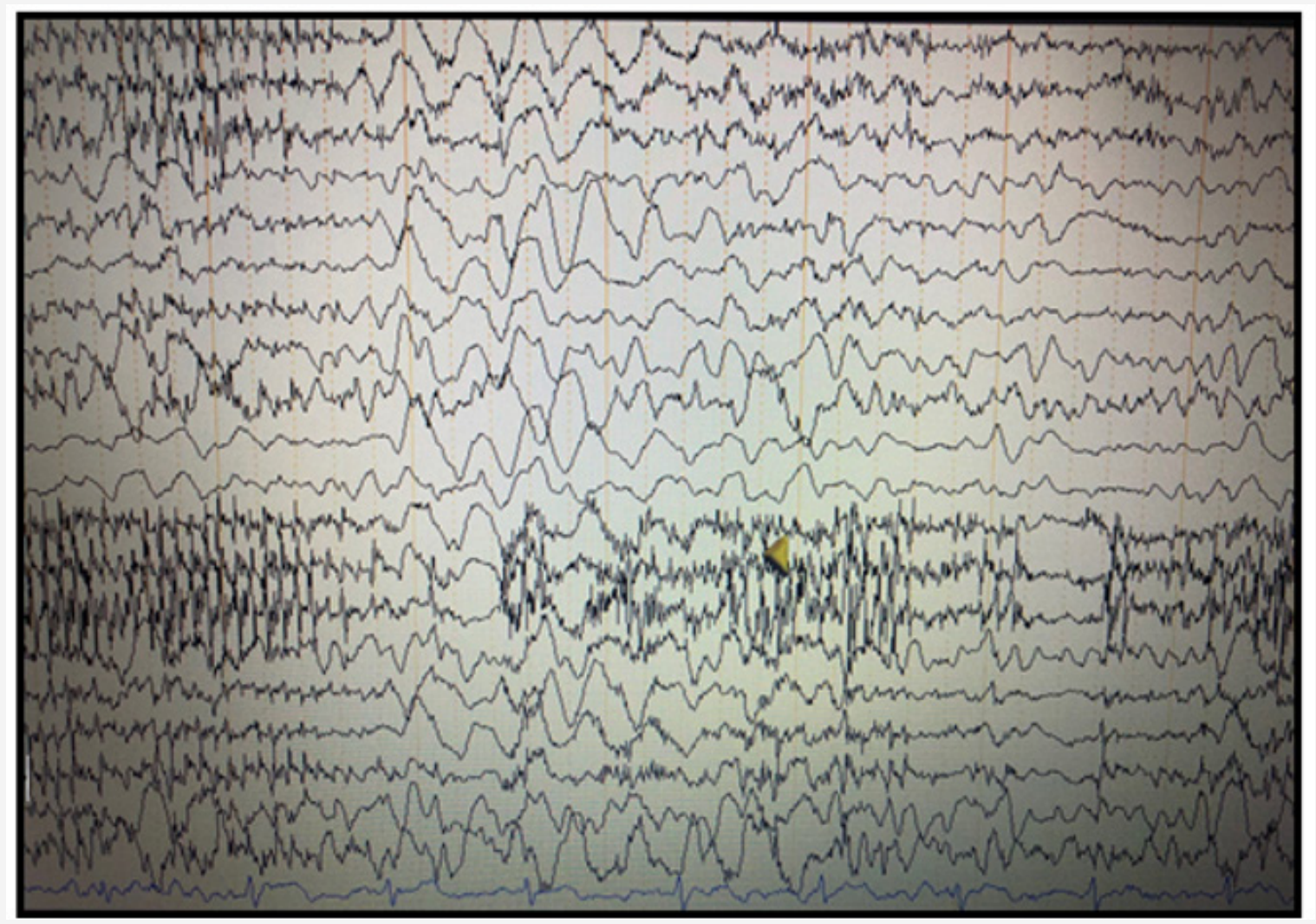

Figure 3

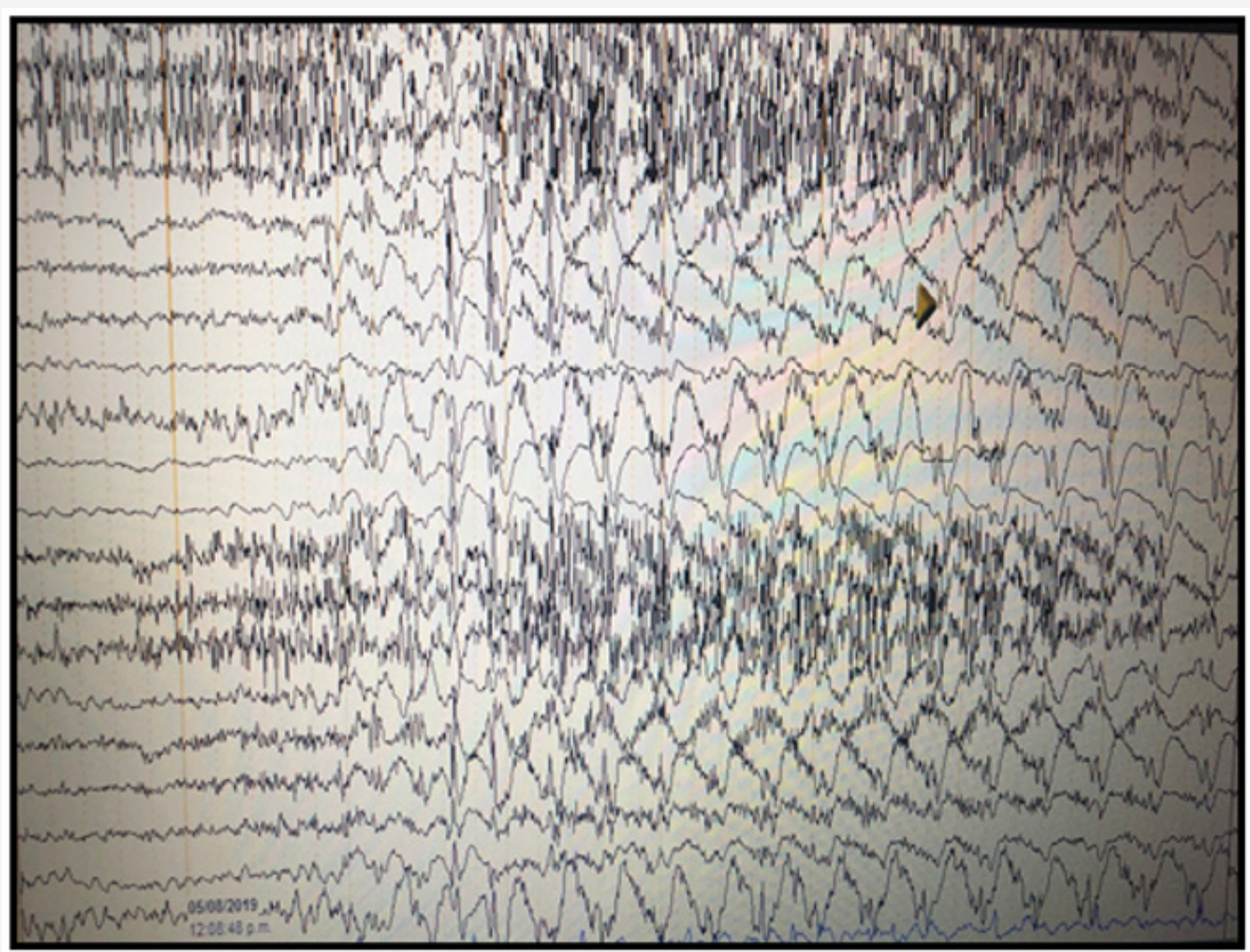

Figure 4 


\section{Treatment}

The basis of treatment is to avoid and suppress triggers such as sleep suppression, fatigue, alcohol intake.

Within the pharmacological treatment the first choice is Valproic acid with a daily dose of $1000 \mathrm{mg}$ or less with an effectiveness of up to $80 \%$. It is suggested to avoid the use of Valproic Acid in women of childbearing age, because significantly, depending on the dose, the risk of fetal malformations increases.

\section{Conclusion}

Juvenile myoclonic epilepsy forms at least one tenth of the epileptic syndromes, of a genetic nature with a family presentation that presents with repetitive abrupt movements predominantly in the upper limb, of a non-progressive nature, its timely detection leads to effectiveness with its first-choice treatment Valproic acid being, knotting the change of lifestyle and its triggers.

\section{Acknowledgement}

None.

\section{Conflict of Interest}

No conflict of interest.

\section{References}

1. Caldera D, Y Burned J (2018) Juvenile Myoclonic Epilepsy. Journal of neuro psychiatry 81(4): 250-256

2. Tirado Requero P (2018) Teen epilepsy. Adolescent 6(1): 44-50. 